\title{
4H-Benzo[d][1,3]oxazin-4-ones and Dihydro Analogs from Substituted Anthranilic Acids and Orthoesters
}

\author{
Joel K. Annor-Gyamfi and Richard A. Bunce * \\ Department of Chemistry, Oklahoma State University, Stillwater, OK 74078-3071, USA; jannorg@okstate.edu \\ * Correspondence: rab@okstate.edu; Tel.: +1-405-744-5952
}

Academic Editor: Andrea Penoni

Received: 14 September 2019; Accepted: 29 September 2019; Published: 1 October 2019

\begin{abstract}
A one-pot route to 2-alkyl and 2-aryl-4H-benzo[d][1,3]oxazin-4-ones (also known as 4H-3,1-benzoxazin-4-ones) has been developed and studied. The method involves the reaction of aryl-substituted anthranilic acids with orthoesters in ethanol catalyzed by acetic acid. Additionally, we have also investigated the reaction under microwave conditions. Not all of the substrates were successful in yielding the target heterocycles as some of the reactions failed to undergo the final elimination. This process led to the isolation of ( \pm )-2-alkyl/aryl-2-ethoxy-1,2-dihydro-4H-benzo[d][1,3]oxazin-4-ones. The formation of the dihydro analogs correlated with the electron density on the aromatic ring: Electron-donating groups favored the $4 \mathrm{H}$ - benzo[d][1,3] oxazin-4-ones, while electron-withdrawing groups tended to favor the dihydro product. Substituting a pyridine ring for the benzene ring in the substrate acid suppressed the reaction.
\end{abstract}

Keywords: $4 H$-benzo[d][1,3]oxazin-4-ones; 1,2-dihydro-4H-benzo[d][1,3]oxazin-4-ones; heterocyclization; anthranilic acid; orthoesters

\section{Introduction}

We recently reported the high-yield synthesis of quinazolin-4(3H)-ones by reaction of 2-aminobenzamides with orthoesters in ethanol promoted by acetic acid [1]. These heterocycles have received considerable attention as they have demonstrated diverse biological activities. With the success of this previous study, we sought to evaluate a similar strategy for the synthesis of 2-alkyl and 2-aryl-4H-benzo[d][1,3]oxazin-4-ones (also known as 4H-3,1-benzoxazin-4-ones). These compounds are less stable, and thus have been less well studied. An early account noted that several substituted structures related to compound 1 exhibited hypolipidemic activity [2]. Other benzoxazinones demonstrated potential as protease inhibitors. In particular, $4 H$-benzo[d][1,3]oxazin-4-one 2 was shown to have substrate inhibitory activity towards the serine protease human leukocyte elastase which is the presumed tissue degenerating agent in the pathogenesis of several diseases [3]. Two additional studies revealed that 3 and 4 interfered with the action of intramembrane serine proteases known as rhomboids that are important in the progression of diseases such as cancer, diabetes, invasion of apicomplexan parasites, and Parkinson's disease [4,5]. Thus, access to these drug candidates would allow further study of the etiology of these diseases and could lead to new drugs to treat them. Structures 1-4 are shown in Figure 1. Apart from investigations of their potential use as drugs, $4 H$-benzo[d][1,3] oxazin-4-ones have also been used as precursors to new heterocycles [6] as well as interesting peptide based oligomers [7].

Classic approaches to $4 H$-benzo[d][1,3] oxazin-4-ones involved reaction of anthranilic acid with two equivalents of benzoyl chloride in pyridine to afford $>90 \%$ of 2-aryl-4H-benzo[d][1,3] oxazin-4-ones [2]. Similarly, treatment of substituted anthranilic acids with one equivalent of various acid chlorides to give the 2-amidobenzoic acid, followed by boiling in acetic anhydride, also afforded these products in good yields $[8,9]$. A later synthesis of $4 H$-benzo[d][1,3]oxazin-4-ones described a high-yield palladium-catalyzed 
cyclocarbonylation of $o$-iodoanilines with acid chlorides [10]. Furthermore, three oxidative approaches to these aryl-fused heterocycles have also been reported. In the first example, copper was used to promote the conversion of 2-alkynylanilines to 2-aryl-4H-benzo[d][1,3]oxazin-4-ones using base conditions under oxygen atmosphere [11]. Another approach utilized oxone in nitromethane at $100{ }^{\circ} \mathrm{C}$ to accomplish the oxidation of 2-arylindoles to these same targets. A third oxidative procedure involved the water assisted oxidation of 2-arylindoles to 2-aryl-4H-benzo[d][1,3]oxazin-4-ones using (diacetoxyiodo)benzene [12]. Finally, a method related to the current procedure was reported for a small series of compounds substituted by phenyl at C2. This procedure utilized microwave energy to promote the formation of 2-phenyl-4H-benzo[d][1,3]oxazin-4-ones from four anthranilic acids and triethyl orthobenzoate [13]. The yields varied in this reaction, but no problems were noted. In the current study, we have attempted to expand the scope of this transformation, examining the reaction of additional aryl-substituted anthranilic acids with four orthoesters. The results of our study are detailed below.
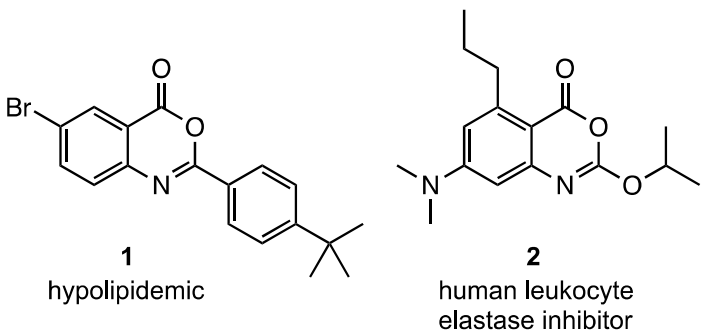<smiles>O=c1oc(OCc2ccccc2)nc2cccc(Cl)c12</smiles>

3

rhomboid protease inhibitors<smiles>O=c1oc(/C=C/c2ccccc2Cl)nc2ccccc12</smiles>

4

Figure 1. 4H-Benzo[d][1,3] oxazin-4-ones with potential drug activity.

\section{Results and Discussion}

Scheme 1 depicts the focus and scope of the current study. We have explored the synthesis of a range of $4 \mathrm{H}$-benzo[d][1,3] oxazin-4-ones from the reaction of six anthranilic acids with a range of commercially available orthoesters (Tables 1 and 2). The reaction was run under two sets of conditions: one under thermal conditions promoted by acid, and the other under microwave conditions. The acid promoted conditions involved heating anthranilic acid ( 1 equivalent) with the orthoester (4.5 equivalents) in the presence of $\mathrm{AcOH}$ (2.6 equivalents), neat, at $100{ }^{\circ} \mathrm{C}$, for $4-48 \mathrm{~h}$. The microwave reaction was run using anthranilic acid (1 equivalent) and the orthoester (2.0-2.7 equivalents), neat, without $\mathrm{AcOH}$, at $100{ }^{\circ} \mathrm{C}$ $(400 \mathrm{~W})$, for $0.75-3 \mathrm{~h}$. Upon cooling, the products from both procedures crystallized from the crude reaction mixtures and were purified by trituration from $5 \%$ pentane in ether.
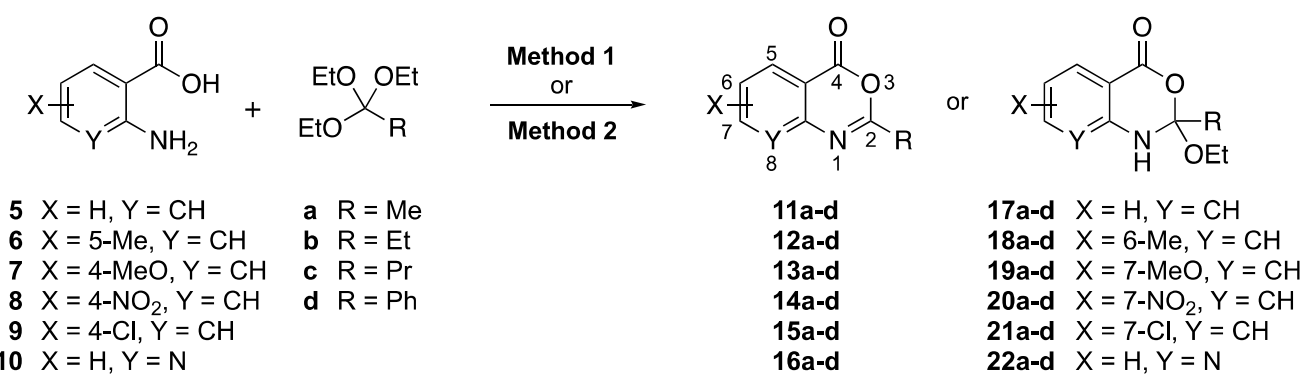

Method 1: anthranilic acid (1 equiv), orthoester (4.5 equiv), $\mathrm{AcOH}$ (2.6 equiv), neat, $100{ }^{\circ} \mathrm{C}, 4-48 \mathrm{~h}$

Method 2: anthranilic acid (1 equiv), orthoester (2.0-2.7 equiv), neat, $\mathrm{MW}, 100^{\circ} \mathrm{C}(400 \mathrm{~W}), 0.75-3 \mathrm{~h}$

Scheme 1. Formation of $4 H$-benzo[d][1,3]oxazin-4-ones and their dihydro analogs from anthranilic acids and orthoesters.

Contrary to what has been reported in related studies to date, we found that not all of the thermal reactions produced the target heterocycles cleanly. In several cases, ( \pm )-2-substituted-1,2-dihydro-4H-benzo[d][1,3]oxazin-4-ones, resulting from ring closure without elimination of the final molecule of ethanol, were isolated. This led us to expand our study to evaluate 
microwave reactions in addition to the standard acid-promoted thermal reactions; however, both protocols afforded the intermediate dihydro compounds from specific substrates. In several cases, it was possible to isolate either product by modification of the procedure. For example, the reaction of anthranilic acid (5) with triethyl orthobenzoate (d) for $24 \mathrm{~h}$ under thermal conditions afforded dihydro product 17d, while extending the reaction to $48 \mathrm{~h}$ yielded $4 H$-benzo[d][1,3]oxazin-4-one 11d. In several other examples, however, it was not possible to induce the final elimination reaction.

Table 1. Formation of $4 H$-benzo[d][1,3] oxazin-4-ones.

\begin{tabular}{|c|c|c|c|c|c|}
\hline Substrate & Product & $\mathbf{R}$ & Method $^{\text {a }}$ & Time (h) & Yield (\%) \\
\hline \multirow[b]{4}{*}{5} & \multirow[b]{4}{*}{$11 a-d$} & \multirow{4}{*}{$\begin{array}{l}\text { a: } \mathrm{Me} \\
\text { b: Et } \\
\text { c: } \mathrm{Pr} \\
\text { d: } \mathrm{Ph}\end{array}$} & $1(2)$ & $48(0.75)$ & $81(82)$ \\
\hline & & & $1(2)$ & $7(0.75)$ & $80(83)$ \\
\hline & & & $1(2)$ & $4(0.75)$ & \multirow[b]{2}{*}{$78(80)$} \\
\hline & & & $1(2)$ & $48(0.75)$ & \\
\hline & \multirow[b]{4}{*}{$12 a-d$} & a: $\mathrm{Me}$ & 2 & 0.75 & \multirow{3}{*}{$\begin{array}{c}68 \\
66 \\
c\end{array}$} \\
\hline & & $\mathrm{b}: \mathrm{Et}$ & 2 & 0.75 & \\
\hline & & c: $\operatorname{Pr}$ & $1(2)$ & $24(0.75)$ & \\
\hline 6 & & $\mathrm{~d}: \mathrm{Ph}$ & 2 & 0.75 & 85 \\
\hline & \multirow[b]{4}{*}{$11 a-d$} & a: $\mathrm{Me}$ & $1(2)$ & $48(0.75)$ & \multirow{4}{*}{$\begin{array}{c}81(82) \\
80(83) \\
b \\
78(80)\end{array}$} \\
\hline & & $\mathrm{b}: \mathrm{Et}$ & $1(2)$ & $7(0.75)$ & \\
\hline & & c: $\operatorname{Pr}$ & $1(2)$ & $4(0.75)$ & \\
\hline 5 & & $\mathrm{~d}: \mathrm{Ph}$ & $1(2)$ & $48(0.75)$ & \\
\hline & \multirow[b]{4}{*}{$13 a-d$} & a: $\mathrm{Me}$ & $1(2)$ & $24(0.75)$ & \multirow{4}{*}{$\begin{array}{l}87(88) \\
86(88) \\
86(87) \\
83(85)\end{array}$} \\
\hline & & $\mathrm{b}: \mathrm{Et}$ & $1(2)$ & $24(0.75)$ & \\
\hline & & c: Pr & $1(2)$ & $24(0.75)$ & \\
\hline 7 & & $\mathrm{~d}: \mathrm{Ph}$ & $1(2)$ & $48(0.75)$ & \\
\hline & \multirow[b]{4}{*}{$14 a-d$} & a: $\mathrm{Me}$ & $1(2)$ & $24(0.75)$ & c \\
\hline & & $\mathrm{b}: \mathrm{Et}$ & $1(2)$ & $24(0.75)$ & c \\
\hline & & c: $\operatorname{Pr}$ & $1(2)$ & $24(0.75)$ & \multirow[b]{2}{*}{91} \\
\hline 8 & & $\mathrm{~d}: \mathrm{Ph}$ & 2 & 3 & \\
\hline & \multirow[b]{4}{*}{$15 a-d$} & a: Me & 2 & 0.75 & \multirow{2}{*}{$\begin{array}{c}85 \\
c\end{array}$} \\
\hline & & $\mathrm{b}: \mathrm{Et}$ & $1(2)$ & $48(0.75)$ & \\
\hline & & c: Pr & $1(2)$ & $48(0.75)$ & c \\
\hline 9 & & $\mathrm{~d}: \mathrm{Ph}$ & 2 & 1.5 & 92 \\
\hline & \multirow[b]{4}{*}{$16 a-d$} & $\mathrm{a}: \mathrm{Me}$ & 1 & 24 & \multirow{3}{*}{ d } \\
\hline & & $\mathrm{b}: \mathrm{Et}$ & 2 & 0.75 & \\
\hline $\mathrm{NH}_{2}$ & & c: $\operatorname{Pr}$ & 2 & 0.75 & \\
\hline 10 & & $\mathrm{~d}: \mathrm{Ph}$ & 2 & 0.75 & $\mathrm{~d}$ \\
\hline
\end{tabular}

${ }^{\text {a }}$ For anthranilic acid (1 equivalent): Method 1: Orthoester (4.5 equivalents), AcOH ( 2.6 equivalents), neat, $100{ }^{\circ} \mathrm{C}$, for the indicated time; Method 2: Orthoester (2.0-2.7 equivalents), neat, $\mathrm{MW}, 10{ }^{\circ} \mathrm{C}$, for the indicated time; ${ }^{\mathrm{b}} \mathrm{An}$ inseparable mixture of the $4 H$-benzo[ $d][1,3]$ oxazin-4-one and the dihydro product was formed; ${ }^{\mathrm{c}}$ The dihydro product formed; ${ }^{\mathrm{d}}$ Only ethyl 2 -aminonicotinate was formed from esterification of the substrate acid. 
Table 2. Formation of ( \pm )-1,2-dihydro-4H-benzo[d][1,3]oxazin-4-ones.

\begin{tabular}{|c|c|c|c|c|c|}
\hline Substrate & Product & $\mathbf{R}$ & Method a & Time (h) & Yield (\%) \\
\hline \multirow{4}{*}{5} & 0 & a: $\mathrm{Me}$ & & & $\mathrm{b}$ \\
\hline & & b: Et & & & $\mathrm{b}$ \\
\hline & & c: $\operatorname{Pr}$ & & & c \\
\hline & 17 & $\mathrm{~d}: \mathrm{Ph}$ & 1 & 24 & 86 \\
\hline \multirow{4}{*}{6} & & a: $\mathrm{Me}$ & 1 & 24 & 88 \\
\hline & & b: Et & 1 & $24(0.75)$ & 87 \\
\hline & & c: $\operatorname{Pr}$ & $1(2)$ & 24 & $88(89)$ \\
\hline & & $\mathrm{d}: \mathrm{Ph}$ & 1 & 48 & 89 \\
\hline \multirow{4}{*}{7} & & a: $\mathrm{Me}$ & & & \\
\hline & & b: Et & & & $\mathrm{b}$ \\
\hline & & c: $\operatorname{Pr}$ & & & b \\
\hline & & $\mathrm{d}: \mathrm{Ph}$ & & & $\mathrm{b}$ \\
\hline \multirow{4}{*}{8} & & a: $\mathrm{Me}$ & $1(2)$ & $24(1.5)$ & $84(87)$ \\
\hline & & b: Et & $1(2)$ & $24(1.5)$ & $86(88)$ \\
\hline & & c: $\operatorname{Pr}$ & $1(2)$ & $24(1.5)$ & $85(88)$ \\
\hline & $20 a-d$ & d: $P h$ & 1 & 24 & 90 \\
\hline \multirow{4}{*}{9} & & a: $\mathrm{Me}$ & 1 & 24 & 84 \\
\hline & & b: Et & $1(2)$ & $48(0.75)$ & $84(86)$ \\
\hline & & c: $\operatorname{Pr}$ & $1(2)$ & $48(0.75)$ & $83(84)$ \\
\hline & $21 a-$ & $\mathrm{d}: \mathrm{Ph}$ & 1 & 48 & 85 \\
\hline \multirow{4}{*}{10} & & a: $\mathrm{Me}$ & 1 & 24 & 85 \\
\hline & & b: Et & & & d \\
\hline & & c: $\operatorname{Pr}$ & & & d \\
\hline & 22a-d & d: $P h$ & & & d \\
\hline
\end{tabular}

${ }^{a}$ For anthranilic acid (1 equivalent): Method 1: Orthoester (4.5 equivalents), $\mathrm{AcOH}$ (2.6 equivalents), neat, $100{ }^{\circ} \mathrm{C}$, for the indicated time; Method 2: Orthoester (2.0-2.7 equivalents), neat, $\mathrm{MW}, 100^{\circ} \mathrm{C}$, for the indicated time; $\mathrm{b}$ Only the $4 H$-benzo[d][1,3]oxazin-4-one product was formed; c An inseparable mixture of the $4 H$-benzo[d][1,3]oxazin-4-one and the dihydro product was formed; d Only ethyl 2-aminonicotinate was formed from esterification of the substrate acid.

The outcome of the reaction appeared to correlate with the electron density in the aromatic ring of the anthranilic acid. For rings substituted with electron donating groups $(\mathrm{OMe}, \mathrm{Me})$ or hydrogen, the $4 H$-benzo[d][1,3] oxazin-4-one product predominated. More electron-poor aromatic rings bearing a second electron-withdrawing group on the aromatic ring $\left(\mathrm{NO}_{2}\right.$ and $\left.\mathrm{Cl}\right)$, in addition to the ortho carbonyl group, showed a tendency to give the 1,2-dihydro-4H-benzo[d][1,3] oxazin-4-one product. Changing the benzene ring of the anthranilic acid to an electron-deficient pyridine, as in 2-aminonicotinic acid (10), almost completely shut down the reaction.

In most cases, it was possible to isolate at least one of the products in pure form. The lone exception was the attempted conversion of 5 to $\mathbf{1 7 c}$ with triethyl orthobutyrate (c), which gave an inseparable mixture of the benzoxazinone and dihydro product under all conditions. Attempts to drive 
the reaction to the benzoxazinone using 5 equivalents of $\mathrm{AcOH}$ or spiking the reaction with $\mathrm{H}_{2} \mathrm{SO}_{4}$ failed to alter the outcome, while treatment with base $\left(\mathrm{K}_{2} \mathrm{CO}_{3}, \mathrm{EtOH}\right.$, molecular sieves) resulted in ring opening to the $\mathrm{N}$-acylanthranilic acid. Finally, in attempted reactions of 2-aminonicotinic acid (10), triethyl orthoacetate (a) proceeded to give dihydro product 21a, but all others afforded only ethyl 2-aminonicotinate resulting from esterification of the substrate acid.

The presumed mechanism for the formation of $4 H$-benzo[d][1,3] oxazin-4-ones is shown in Scheme 2. The process involves initial protonation of the orthoester and loss of ethanol to afford the stabilized carbocation A. Attack on A by the anthranilic acid amino group, proton exchange and loss of a second molecule of ethanol would then yield iminium ion B. Subsequent closure of the acid oxygen on the iminium carbon and proton exchange would give ring closed dihydro product $\mathbf{C}$. Finally, protonation of $\mathbf{C}$ to give $\mathbf{D}$ and loss of ethanol would then generate the desired heterocycle $\mathbf{E}$.

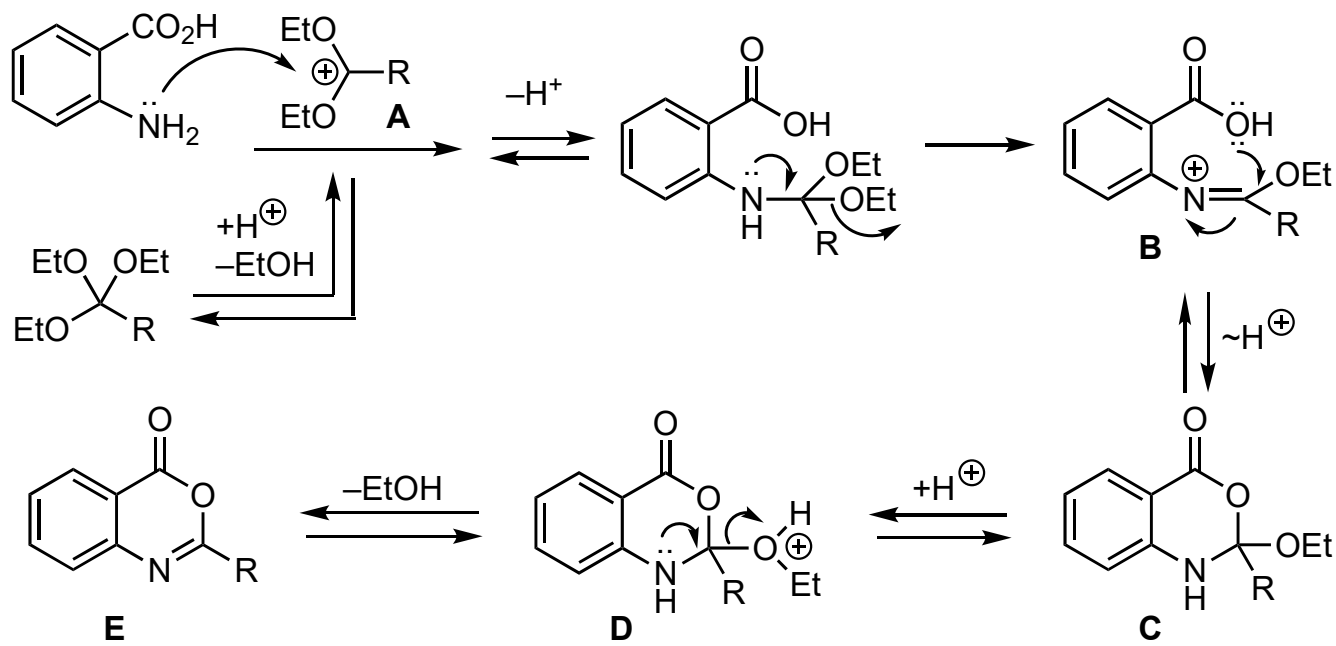

Scheme 2. Presumed mechanism for formation of $4 H$-benzo[d][1,3]oxazin-4-ones.

Difficulty was noted in the final elimination of ethanol when the aromatic ring carried a second electron-withdrawing group (EWG) in addition to the adjacent ester carbonyl. For this elimination, the availability of the unshared electron pair on N1 and its ability to align antiperiplanar to the departing alcohol were assumed to be keys to the success of this elimination. The availability of these electrons, however, would be diminished in substrates with a strongly electron-deficient ring. Relatively little seems to be known about the necessity for the antiperiplanar alignment of the unshared pair for efficient elimination in this system. This anomeric effect has been extensively studied in acetals and hemiacetals [14], but much less so for their nitrogen analogs since elimination is normally facile. If achievable, conformation D1 would result in good overlap for elimination of ethanol (see Figure 2). Placement of the C2 ethoxy group in a pseudo-axial position for this elimination should be favorable since this group is considered to be sterically smaller than an alkyl group $[15,16]$. However, this conformation also maximizes overlap of the N1 electron pair with the aromatic ring as well as the ortho carbonyl, and this could slow the elimination if the aromatic ring bears an additional electron-withdrawing substituent. The alternative conformation D2 is not optimally aligned for elimination (see Figure 2). If the ring closure and protonation steps led to this conformation, conversion to D1 could occur, but would likely be sluggish since flexibility in this rigid system is allowed only at the saturated atoms of the benzoxazinone. 


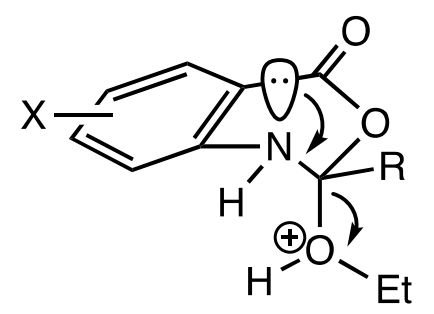

D1

- favorable alignment of the $\mathrm{N}^{-}$ pair with the $\mathrm{EtOH}$ leaving group

- the $\mathrm{e}^{-}$pair is less available when $\mathrm{X}=\mathrm{EWG}$
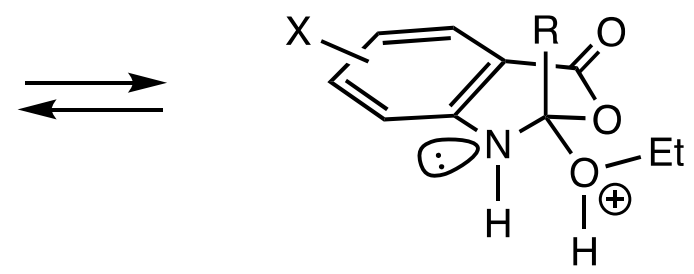

D2

- unfavorable alignment of the $\mathrm{N} \mathrm{e}^{-}$ pair with the EtOH leaving group

- the $e^{-}$pair has less overlap with the adjacent aryl ring

Figure 2. Conformational options for intermediate D.

\section{Experimental Section}

\subsection{General}

All commercial reagents and solvents were used as received. Unless otherwise indicated, all reactions were carried out under dry $\mathrm{N}_{2}$ in oven-dried glassware. Reactions were monitored by thin layer chromatography (TLC) using silica gel GF plates (Analtech No. 21521, Newark, DE, USA). Microwave reactions were performed in 2-5 mL microwave tubes (Biotage No. 354624, Charlotte, $\mathrm{NC}$, USA) at $100{ }^{\circ} \mathrm{C}$ using a Biotage Initiator 2.5 system (Charlotte, NC, USA) at a power of $400 \mathrm{~W}$ from a magneton at $2.45 \mathrm{GHz}$. Melting points were obtained on a MEL-TEMP apparatus (Laboratory Devices, Cambridge, MA, USA), and were uncorrected. FT-IR spectra were run using a Varian Scimitar FTS 800 spectrophotometer (Randolph, MA, USA) as thin films or nujol mulls on $\mathrm{NaCl}$ disks. ${ }^{1} \mathrm{H}-$ and ${ }^{13} \mathrm{C}-\mathrm{NMR}$ spectra were measured using a Bruker Avance 400 system (Billerica, MA, USA) in the indicated solvents (Cambridge Isotope, Andover, MA, USA) at $400 \mathrm{MHz}$ and $101 \mathrm{MHz}$, respectively, with $\left(\mathrm{CH}_{3}\right)_{4} \mathrm{Si}$ as the internal standard; coupling constants $(J)$ are given in $\mathrm{Hz}$. Copies of NMR spectra for the products are included in the Supplementary Information. High-resolution mass spectra (HRMS-ESI) were obtained using a Thermo LTQ-Orbitrap XL mass spectrometer (Thermo Scientific, Waltham, MA, USA).

\subsection{Representative Procedure under Thermal Conditions. Method 1}

The anthranilic acid $(1.0 \mathrm{mmol})$ and the orthoester $(4.5 \mathrm{mmol})$ were placed in a $15-\mathrm{mL}$ Chemglass screw-cap pressure tube (CG-1880-01, Vineland, NJ, USA). Glacial acetic acid (2.6 mmol) was added, $\mathrm{N}_{2}$ was introduced to the vessel and the cap was tightened. The vessel was heated at $100{ }^{\circ} \mathrm{C}$ under neat conditions for $4-48 \mathrm{~h}$, and then cooled to room temperature. Upon cooling, the crude product crystallized from the reaction mixture and was purified by trituration from $5 \%$ ether in pentane.

\subsection{Representative Procedure under Microwave Conditions. Method 2}

A solution of the anthranilic acid $(1.0 \mathrm{mmol})$ in the orthoester $(0.45 \mathrm{~mL}, 2.0-2.7$ equivalents $)$ was prepared in a $5-\mathrm{mL}$ microwave tube and stirred for $30 \mathrm{~s}$ prior to irradiation at the "high absorption" setting. The reaction was performed under $\mathrm{N}_{2}$ at $100{ }^{\circ} \mathrm{C}(400 \mathrm{~W})$ for $0.75-3 \mathrm{~h}$. Upon cooling, the crude product crystallized from the reaction mixture and was purified by trituration from $5 \%$ ether in pentane.

The following compounds were prepared:

2-Methyl-4H-benzo[d][1,3]oxazin-4-one (11a): $130 \mathrm{mg}(81 \%$, method 1$)$ and $132 \mathrm{mg}(82 \%$, method 2$)$ as white crystals, m.p. $79-80{ }^{\circ} \mathrm{C}$ (ref. [8] 80-81 ${ }^{\circ} \mathrm{C}$ ); IR (thin film): $1700,1624 \mathrm{~cm}^{-1} ;{ }^{1} \mathrm{H}-\mathrm{NMR}(400 \mathrm{MHz}$, $\left.\mathrm{CDCl}_{3}\right): \delta 8.29(\mathrm{dd}, 1 \mathrm{H}, J=7.5,1.6 \mathrm{~Hz}, \mathrm{ArH}), 7.78(\mathrm{ddd}, 1 \mathrm{H}, J=8.4,7.1,1.6 \mathrm{~Hz}, \mathrm{ArH}), 7.69(\mathrm{~d}, 1 \mathrm{H}$, $J=8.4 \mathrm{~Hz}, \mathrm{ArH}), 7.48(\mathrm{ddd}, 1 \mathrm{H}, J=8.1,7.1,1.2 \mathrm{~Hz}, \mathrm{ArH}), 2.60\left(\mathrm{~s}, 3 \mathrm{H}, \mathrm{CH}_{3}\right) ;{ }^{13} \mathrm{C}-\mathrm{NMR}(101 \mathrm{MHz}$, 
$\mathrm{CDCl}_{3}$ ): $\delta 164.1,153.2,149.4,134.9,127.0,126.5,126.3,120.3,22.2 ; \mathrm{HRMS}(\mathrm{ESI}): \mathrm{m} / z[\mathrm{M}+\mathrm{H}]^{+}$calcd. for $\mathrm{C}_{9} \mathrm{H}_{7} \mathrm{NO}_{2}: 162.0555$, found: 162.0553 .

2-Ethyl-4H-benzo[d][1,3]oxazin-4-one (11b): $140 \mathrm{mg}$ (80\%, method 1) and $145 \mathrm{mg}(83 \%$, method 2) as white crystals, m.p. $83-84{ }^{\circ} \mathrm{C}$ (ref. [8] m.p. $85-86{ }^{\circ} \mathrm{C}$ ); IR (thin film): $1750,1645 \mathrm{~cm}^{-1} ;{ }^{1} \mathrm{H}-\mathrm{NMR}$ $\left(400 \mathrm{MHz}, \mathrm{CDCl}_{3}\right): \delta 8.20(\mathrm{dd}, 1 \mathrm{H}, J=7.9,1.5 \mathrm{~Hz}, \mathrm{ArH}), 7.80(\mathrm{ddd}, 1 \mathrm{H}, J=8.4,7.5,1.5 \mathrm{~Hz}, \mathrm{ArH}), 7.57(\mathrm{~d}$, $1, J=8.4 \mathrm{~Hz}, \mathrm{ArH}), 7.50(\mathrm{ddd}, 1 \mathrm{H}, J=8.2,7.5,1.2 \mathrm{~Hz}, \mathrm{ArH}), 2.73\left(\mathrm{q}, 2 \mathrm{H}, J=7.5 \mathrm{~Hz}, \mathrm{CH}_{2} \mathrm{CH}_{3}\right), 1.37(\mathrm{t}$, $\left.3 \mathrm{H}, J=7.5 \mathrm{~Hz}, \mathrm{CH}_{2} \mathrm{CH}_{3}\right) ;{ }^{13} \mathrm{C}-\mathrm{NMR}\left(101 \mathrm{MHz}, \mathrm{CDCl}_{3}\right): \delta 163.9,159.9,146.5,136.5,128.4,128.1,126.6$, 116.9, 28.2, 10.3; HRMS (ESI): $m / z[\mathrm{M}+\mathrm{H}]^{+}$calcd. for $\mathrm{C}_{10} \mathrm{H}_{9} \mathrm{NO}_{2}: 176.0712$, found: 176.0713.

2-Phenyl-4H-benzo[d][1,3]oxazin-4-one (11d): $174 \mathrm{mg}(78 \%$, method 1$)$ and $179 \mathrm{mg}(80 \%$, method 2$)$ as off-white crystals, m.p. $121-122{ }^{\circ} \mathrm{C}$ (ref. [8] m.p. $123{ }^{\circ} \mathrm{C}$ ); IR (thin film): $1762,1615 \mathrm{~cm}^{-1} ;{ }^{1} \mathrm{H}-\mathrm{NMR}$ $\left(400 \mathrm{MHz}, \mathrm{CDCl}_{3}\right)$ : $\delta 8.34-8.30$ (complex, $\left.2 \mathrm{H}, \mathrm{ArH}\right), 8.25(\mathrm{dd}, 1 \mathrm{H}, J=7.9,1.5, \mathrm{ArH}), 7.84(\mathrm{ddd}, 1 \mathrm{H}$, $J=8.1,7.3,1.5 \mathrm{~Hz}, \mathrm{ArH}), 7.70(\mathrm{~d}, J=8.1 \mathrm{~Hz}, \mathrm{ArH}), 7.62-7.48$ (complex, 4H, ArH); ${ }^{13} \mathrm{C}-\mathrm{NMR}(101 \mathrm{MHz}$, $\mathrm{CDCl}_{3}$ ): $\delta 159.6,157.1,147.0,136.6,132.6,130.3,128.8,128.6,128.32,128.26,127.2,117.0$; HRMS (ESI): $\mathrm{m} / \mathrm{z}[\mathrm{M}+\mathrm{H}]^{+}$calcd. for $\mathrm{C}_{14} \mathrm{H}_{9} \mathrm{NO}_{2}: 224.0712$, found: 224.0709 .

2,6-Dimethyl-4H-benzo[d][1,3]oxazin-4-one (12a): $119 \mathrm{mg}(68 \%$, method 2) as off-white crystals, m.p. 122-123 ${ }^{\circ} \mathrm{C}$; IR (thin film): 1682, $1595 \mathrm{~cm}^{-1} ;{ }^{1} \mathrm{H}-\mathrm{NMR}\left(400 \mathrm{MHz}, \mathrm{CDCl}_{3}\right): \delta 7.98(\mathrm{~s}, 1 \mathrm{H}, \mathrm{ArH}), 7.61(\mathrm{~d}$, $1 \mathrm{H}, J=8.2 \mathrm{~Hz}, \mathrm{ArH}), 7.44(\mathrm{~d}, 1 \mathrm{H}, J=8.2 \mathrm{~Hz}, \mathrm{ArH}), 2.47\left(\mathrm{~s}, 3 \mathrm{H}, \mathrm{CH}_{3}\right), 2.45\left(\mathrm{~s}, 3 \mathrm{H}, \mathrm{CH}_{3}\right) ;{ }^{13} \mathrm{C}-\mathrm{NMR}$ (101 MHz, $\mathrm{CDCl}_{3}$ ): $\delta$ 159.9, 159.4, 144.3, 138.5, 137.7, 128.0, 126.2, 116.3, 21.3, 21.2; HRMS (ESI): $\mathrm{m} / z$ [M $+\mathrm{H}]^{+}$calcd. for $\mathrm{C}_{10} \mathrm{H}_{9} \mathrm{NO}_{2}$ : 176.0712, found: 176.0709 .

2-Ethyl-6-methyl-4H-benzo[d][1,3]oxazin-4-one (12b): $125 \mathrm{mg}$ (66\%, method 2) as white crystals, m.p. 103-104 ${ }^{\circ} \mathrm{C}$; IR (thin film): 1686, $1596 \mathrm{~cm}^{-1} ;{ }^{1} \mathrm{H}-\mathrm{NMR}\left(400 \mathrm{MHz}, \mathrm{CDCl}_{3}\right): \delta 7.98$ (s, $\left.1 \mathrm{H}, \mathrm{ArH}\right), 7.60$ $(\mathrm{d}, 1 \mathrm{H}, J=8.2 \mathrm{~Hz}, \mathrm{ArH}), 7.46(\mathrm{~d}, 1 \mathrm{H}, J=8.2 \mathrm{~Hz}, \mathrm{ArH}), 2.71\left(\mathrm{q}, 2 \mathrm{H}, J=7.5 \mathrm{~Hz}, \mathrm{CH}_{2} \mathrm{CH}_{3}\right), 2.47(\mathrm{~s}, 3 \mathrm{H}$, $\left.\mathrm{ArCH}_{3}\right), 1.36\left(\mathrm{t}, 3 \mathrm{H}, J=7.5 \mathrm{~Hz}, \mathrm{CH}_{2} \mathrm{CH}_{3}\right) ;{ }^{13} \mathrm{C}-\mathrm{NMR}\left(101 \mathrm{MHz}, \mathrm{CDCl}_{3}\right): \delta 163.1,160.1,144.3,138.4$, 137.7, 128.0, 126.4, 116.5, 26.1, 21.2, 10.3; HRMS (ESI): $\mathrm{m} / z[\mathrm{M}+\mathrm{H}]^{+}$calcd. for $\mathrm{C}_{11} \mathrm{H}_{11} \mathrm{NO}_{2}: 190.0868$, found: 190.0865 .

6-Methyl-2-phenyl-4H-benzo[d][1,3]oxazin-4-one (12d): $202 \mathrm{mg}$ (85\%, method 2) as off-white crystals, m.p. 144-145 ${ }^{\circ} \mathrm{C}$; IR (thin film): 1752, $1607 \mathrm{~cm}^{-1} ;{ }^{1} \mathrm{H}-\mathrm{NMR}\left(400 \mathrm{MHz}, \mathrm{CDCl}_{3}\right): \delta 8.29$ (d, $\left.2 \mathrm{H}, J=8.2 \mathrm{~Hz}, \mathrm{ArH}\right)$, $8.04(\mathrm{~s}, 1 \mathrm{H}, \mathrm{ArH}), 7.66-7.47$ (complex, 5H, ArH), 2.49 (s, 3H, $\left.\mathrm{ArCH}_{3}\right) ;{ }^{13} \mathrm{C}-\mathrm{NMR}\left(101 \mathrm{MHz}, \mathrm{CDCl}_{3}\right)$ : $\delta$ $159.8,156.4,144.8,138.7,137.8,132.4,130.4,128.7,128.19,128.17,127.0,116.7,21.2 ;$ HRMS (ESI): $m / z$ [M $+\mathrm{H}]^{+}$for $\mathrm{C}_{15} \mathrm{H}_{11} \mathrm{NO}_{2}: 238.0868$, found: 238.0870 .

7-Methoxy-2-methyl-4H-benzo[d][1,3]oxazin-4-one (13a): $166 \mathrm{mg}(87 \%$, method 1) and $168 \mathrm{mg}(88 \%$, method 2) as light yellow crystals, m.p. $119-120{ }^{\circ} \mathrm{C}$; IR (nujol): 2830, 1747, $1610 \mathrm{~cm}^{-1}$; ${ }^{1} \mathrm{H}-\mathrm{NMR}$ $\left(400 \mathrm{MHz}\right.$, DMSO- $\left.d_{6}\right): \delta 8.00(\mathrm{~d}, 1 \mathrm{H}, J=8.8 \mathrm{~Hz}, \mathrm{ArH}), 7.14(\mathrm{dd}, 1 \mathrm{H}, J=8.8,2.5 \mathrm{~Hz}, \mathrm{ArH}), 7.05$ (d, $1 \mathrm{H}, J=2.5 \mathrm{~Hz}, \mathrm{ArH}), 3.91\left(\mathrm{~s}, 3 \mathrm{H}, \mathrm{OCH}_{3}\right), 2.38\left(\mathrm{~s}, 3 \mathrm{H}, \mathrm{CH}_{3}\right) ;{ }^{13} \mathrm{C}-\mathrm{NMR}\left(101 \mathrm{MHz}, \mathrm{DMSO}-d_{6}\right): \delta 166.3$, 161.5, 159.2, 149.0, 130.2, 117.1, 109.5, 109.1, 56.5, 21.5; HRMS (ESI): $m / z[\mathrm{M}+\mathrm{H}]^{+}$calcd. for $\mathrm{C}_{10} \mathrm{H}_{9} \mathrm{NO}_{3}$ : 192.0661, found: 192.0659 .

2-Ethyl-7-methoxy-4H-benzo[d][1,3]oxazin-4-one (13b): $176 \mathrm{mg}$ (86\%, method 1) and $181 \mathrm{mg}(88 \%$, method 2) as light yellow crystals, m.p. $89-90^{\circ} \mathrm{C}$; IR (nujol): $2840,1767,1644,1609 \mathrm{~cm}^{-1} ;{ }^{1} \mathrm{H}-\mathrm{NMR}(400 \mathrm{MHz}$, DMSO- $\left.d_{6}\right): \delta 7.99(\mathrm{~d}, 1 \mathrm{H}, J=8.8 \mathrm{~Hz}, \mathrm{ArH}) 7.14(\mathrm{dd}, 1 \mathrm{H}, J=8.8,2.5 \mathrm{~Hz}, \mathrm{ArH}), 7.07(\mathrm{~d}, 1 \mathrm{H}, J=2.5 \mathrm{~Hz}$, $\mathrm{ArH}), 3.92\left(\mathrm{~s}, 3 \mathrm{H}, \mathrm{OCH}_{3}\right), 2.68\left(\mathrm{q}, 2 \mathrm{H}, J=7.5 \mathrm{~Hz}, \mathrm{CH}_{2} \mathrm{CH}_{3}\right), 1.24\left(\mathrm{t}, 3 \mathrm{H}, J=7.5 \mathrm{~Hz}, \mathrm{CH}_{2} \mathrm{CH}_{3}\right) ;{ }^{13} \mathrm{C}-\mathrm{NMR}$ (101 MHz, DMSO- $\left.d_{6}\right): \delta 166.3,164.9,159.2,149.0,130.1,117.3,109.7,109.1,56.6,27.7,10.2$; HRMS (ESI): $\mathrm{m} / \mathrm{z}[\mathrm{M}+\mathrm{H}]^{+}$calcd. for $\mathrm{C}_{11} \mathrm{H}_{11} \mathrm{NO}_{3}: 206.0817$, found: 206.0817.

7-Methoxy-2-propyl-4H-benzo[d][1,3]oxazin-4-one (13c): $189 \mathrm{mg}$ (86\%, method 1) and $191 \mathrm{mg}(87 \%$, method 2) as light yellow crystals, m.p. $77-78^{\circ} \mathrm{C}$; IR (nujol): 2841, 1766, 1643, $1609 \mathrm{~cm}^{-1}$; ${ }^{1} \mathrm{H}-\mathrm{NMR}$ $\left(400 \mathrm{MHz}, \mathrm{DMSO}-d_{6}\right): \delta 8.00(\mathrm{~d}, 1 \mathrm{H}, J=8.8 \mathrm{~Hz}, \mathrm{ArH}), 7.14(\mathrm{dd}, 1 \mathrm{H}, J=8.8,2.5 \mathrm{~Hz}, \mathrm{ArH}), 7.07(\mathrm{~d}, 1 \mathrm{H}$, $J=2.5 \mathrm{~Hz}, \mathrm{ArH}), 3.92\left(\mathrm{~s}, 3 \mathrm{H}, \mathrm{OCH}_{3}\right), 2.63\left(\mathrm{t}, 2 \mathrm{H}, J=7.4 \mathrm{~Hz}, \mathrm{CH}_{2} \mathrm{CH}_{2} \mathrm{CH}_{3}\right), 1.75$ (sextet, $2 \mathrm{H}, J=7.4 \mathrm{~Hz}$, $\left.\mathrm{CH}_{2} \mathrm{CH}_{2} \mathrm{CH}_{3}\right) ; 0.98\left(\mathrm{t}, 3 \mathrm{H}, J=7.4 \mathrm{~Hz}, \mathrm{CH}_{2} \mathrm{CH}_{2} \mathrm{CH}_{3}\right) ;{ }^{13} \mathrm{C}-\mathrm{NMR}\left(101 \mathrm{MHz}\right.$, DMSO- $\left.d_{6}\right): \delta 166.3,163.9$, 
159.2, 148.9, 130.1, 117.3, 109.7, 109.1, 56.6, 36.2, 19.2, 13.9; HRMS (ESI): $m / z[M+\mathrm{H}]^{+}$calcd. for $\mathrm{C}_{12} \mathrm{H}_{13} \mathrm{NO}_{3}: 220.0974$, found: 220.0971.

7-Methoxy-2-phenyl-4H-benzo[d][1,3]oxazin-4-one (13d): $210 \mathrm{mg}$ (83\%, method 1) and $215 \mathrm{mg}(85 \%$, method 2) as light yellow crystals, m.p. $149-150{ }^{\circ} \mathrm{C}$; IR (nujol): $2842,1744,1607 \mathrm{~cm}^{-1} ;{ }^{1} \mathrm{H}-\mathrm{NMR}$ $\left(400 \mathrm{MHz}, \mathrm{DMSO}-d_{6}\right): \delta 8.17(\mathrm{~d}, 2 \mathrm{H}, J=8.1 \mathrm{~Hz}, \mathrm{ArH}), 8.03(\mathrm{~d}, 1 \mathrm{H}, J=9.4 \mathrm{~Hz}, \operatorname{ArH}), 7.67(\mathrm{~d}, 1 \mathrm{H}$, $J=7.3 \mathrm{~Hz}, \mathrm{ArH}), 7.59(\mathrm{t}, 2 \mathrm{H}, J=8.1 \mathrm{~Hz}, \mathrm{ArH}), 7.15(\mathrm{~m}, 2 \mathrm{H}, \mathrm{ArH}), 3.94\left(\mathrm{~s}, 3 \mathrm{H}, \mathrm{OCH}_{3}\right) ;{ }^{13} \mathrm{C}-\mathrm{NMR}$ $\left(101 \mathrm{MHz}, \mathrm{DMSO}-d_{6}\right): \delta 166.4,158.8,157.6,149.2,133.2,130.5,130.3,129.4,128.3,117.6,109.9,109.6$, 56.6; HRMS (ESI): $\mathrm{m} / \mathrm{z}[\mathrm{M}+\mathrm{H}]^{+}$calcd. for $\mathrm{C}_{15} \mathrm{H}_{11} \mathrm{NO}_{3}: 254.0817$, found: 254.0814 .

7-Nitro-2-phenyl-4H-benzo[d][1,3]oxazin-4-one (14d): $244 \mathrm{mg}(91 \%$, method 2) as light orange crystals, m.p. $175-176{ }^{\circ} \mathrm{C}$; IR (thin film): 1757, 1629, $1608,1530,1349 \mathrm{~cm}^{-1} ;{ }^{1} \mathrm{H}-\mathrm{NMR}\left(400 \mathrm{MHz}, \mathrm{CDCl}_{3}\right): \delta 8.52$ $(\mathrm{d}, 1 \mathrm{H}, J=2.2 \mathrm{~Hz}, \mathrm{ArH}), 8.41(\mathrm{~d}, 1 \mathrm{H}, J=8.6 \mathrm{~Hz}, \mathrm{ArH}), 8.33(\mathrm{~d}, 2 \mathrm{H}, J=7.4 \mathrm{~Hz}, \mathrm{ArH}), 8.29(\mathrm{dd}, 1 \mathrm{H}, J=8.6$, $2.2 \mathrm{~Hz}, \mathrm{ArH}), 7.64(\mathrm{t}, 1 \mathrm{H}, J=7.4 \mathrm{~Hz}, \mathrm{ArH}), 7.55(\mathrm{t}, 2 \mathrm{H}, J=7.4 \mathrm{~Hz}, \mathrm{ArH}) ;{ }^{13} \mathrm{C}-\mathrm{NMR}\left(101 \mathrm{MHz}, \mathrm{CDCl}_{3}\right): \delta$ 159.0, 157.9, 152.9, 148.1, 133.6, 130.4, 129.3, 129.0, 128.7, 122.5, 122.0, 121.3; HRMS (ESI): $m / z$ [M + H] ${ }^{+}$ calcd. for $\mathrm{C}_{14} \mathrm{H}_{8} \mathrm{~N}_{2} \mathrm{O}_{4}$ : 269.0562, found: 269.0558 .

7-Chloro-2-methyl-4H-benzo[d][1,3]oxazin-4-one (15a): $166 \mathrm{mg}$ (85\%, method 2) as white crystals, m.p. 149-150 ${ }^{\circ} \mathrm{C}$; IR (thin film): 1762, 1696, 1642, $1596 \mathrm{~cm}^{-1} ;{ }^{1} \mathrm{H}-\mathrm{NMR}\left(400 \mathrm{MHz}, \mathrm{CDCl}_{3}\right): \delta 8.11(\mathrm{~d}, 1 \mathrm{H}$, $J=8.4 \mathrm{~Hz}, \mathrm{ArH}), 7.54(\mathrm{~d}, 1 \mathrm{H}, J=2.0 \mathrm{~Hz}, \mathrm{ArH}), 7.46(\mathrm{dd}, 1 \mathrm{H}, J=8.4,2.0 \mathrm{~Hz}, \mathrm{ArH}), 2.47\left(\mathrm{~s}, 3 \mathrm{H} \mathrm{CH}_{3}\right)$; ${ }^{13} \mathrm{C}-\mathrm{NMR}\left(101 \mathrm{MHz}, \mathrm{CDCl}_{3}\right): \delta 161.6,158.9,147.5,142.9,129.7,128.8,126.3,115.1,21.4$; HRMS (ESI): $m / z[\mathrm{M}+\mathrm{H}]^{+}$calcd. for $\mathrm{C}_{9} \mathrm{H}_{6}{ }^{35} \mathrm{ClNO}_{2}:$ 196.0165, found: 196.0166 .

7-Chloro-2-phenyl-4H-benzo[d][1,3]oxazin-4-one (15d): $237 \mathrm{mg}$ (92\%, method 2) as off-white crystals, m.p. 188-189 ${ }^{\circ} \mathrm{C}$; IR (thin film): 1760, 1619, $1596 \mathrm{~cm}^{-1} ;{ }^{1} \mathrm{H}-\mathrm{NMR}\left(400 \mathrm{MHz}, \mathrm{CDCl}_{3}\right): \delta 8.31$ (d, 2H, J = $7.4 \mathrm{~Hz}$, $\mathrm{ArH}), 8.17(\mathrm{~d}, 1 \mathrm{H}, J=8.4 \mathrm{~Hz}, \mathrm{ArH}), 7.70(\mathrm{~d}, 1 \mathrm{H}, J=2.0 \mathrm{~Hz}, \mathrm{ArH}), 7.60(\mathrm{tt}, 1 \mathrm{H}, J=7.4,1.4 \mathrm{~Hz}, \mathrm{ArH}), 7.52$ $(\mathrm{t}, 2 \mathrm{H}, J=7.4 \mathrm{~Hz}, \mathrm{ArH}), 7.48(\mathrm{dd}, 1 \mathrm{H}, J=8.4,2.0 \mathrm{~Hz}, \mathrm{ArH}) ;{ }^{13} \mathrm{C}-\mathrm{NMR}\left(101 \mathrm{MHz}, \mathrm{CDCl}_{3}\right): \delta 158.8,158.3$, $148.1,143.0,133.0,129.9,129.86,128.83,128.79,128.5,127.0,115.4 ;$ HRMS (ESI): $m / z[M+H]^{+}$calcd. for $\mathrm{C}_{14} \mathrm{H}_{8}{ }^{35} \mathrm{ClNO}_{2}: 258.0322$, found: 258.0317 .

( \pm )-2-Ethoxy-2-phenyl-1,2-dihydro-4H-benzo[d][1,3]oxazin-4-one (17d): $232 \mathrm{mg}$ (86\%, method 1) as white crystals, m.p. $92-93{ }^{\circ} \mathrm{C}$; IR (thin film): $3254,1668,1595 \mathrm{~cm}^{-1},{ }^{1} \mathrm{H}-\mathrm{NMR}\left(400 \mathrm{MHz}, \mathrm{CDCl}_{3}\right): \delta 12.09$ (br s, $1 \mathrm{H}, \mathrm{NH}), 8.94(\mathrm{dd}, 1 \mathrm{H}, J=8.5,1.1 \mathrm{~Hz}), \mathrm{ArH}), 8.1(\mathrm{dd}, 1 \mathrm{H}, J=8.0,1.7 \mathrm{~Hz}, \mathrm{ArH}), 8.06(\mathrm{dd}, 2 \mathrm{H}$, $J=7.8,1.5 \mathrm{~Hz}, \mathrm{ArH}), 7.64-7.49$ (complex, 4H, ArH), $7.13(\mathrm{td}, 1 \mathrm{H}, J=7.4,1.2 \mathrm{~Hz}, \mathrm{ArH}), 4.43(\mathrm{q}, 2 \mathrm{H}$, $\left.J=7.1 \mathrm{~Hz}, \mathrm{OCH}_{2} \mathrm{CH}_{3}\right), 1.43\left(\mathrm{t}, 3 \mathrm{H}, J=7.1 \mathrm{~Hz}, \mathrm{OCH}_{2} \mathrm{CH}_{3}\right) ;{ }^{13} \mathrm{C}-\mathrm{NMR}\left(101 \mathrm{MHz}, \mathrm{CDCl}_{3}\right): \delta 168.6,165.4$, $141.9,134.9,134.7,131.9,130.9,128.8,127.4,122.6,120.5,115.5,61.5,14.2$; HRMS (ESI): $m / z[\mathrm{M}+\mathrm{H}]^{+}$ calcd. for $\mathrm{C}_{16} \mathrm{H}_{15} \mathrm{NO}_{2}$ : 254.1181, found: 254.1177 .

( \pm )-2-Ethoxy-2,6-dimethyl-1,2-dihydro-4H-benzo[d][1,3]oxazin-4-one (18a): $195 \mathrm{mg}(88 \%$, method 1) as white crystals, m.p. $110-11{ }^{\circ} \mathrm{C}$; IR (thin film): $3266,1677,1595 \mathrm{~cm}^{-1},{ }^{1} \mathrm{H}-\mathrm{NMR}\left(400 \mathrm{MHz}, \mathrm{CDCl}_{3}\right): \delta$ $10.97(\mathrm{br} \mathrm{s}, 1 \mathrm{H}, \mathrm{NH}), 8.57(\mathrm{~d}, 1 \mathrm{H}, J=8.6 \mathrm{~Hz}, \mathrm{ArH}), 7.83(\mathrm{~s}, 1 \mathrm{H}, \mathrm{ArH}), 7.34(\mathrm{~d}, 1 \mathrm{H}, J=8.6 \mathrm{~Hz}, \mathrm{ArH}), 4.37$ $\left(\mathrm{q}, 2 \mathrm{H}, J=7.1 \mathrm{~Hz}, \mathrm{OCH}_{2} \mathrm{CH}_{3}\right), 2.33\left(\mathrm{~s}, 3 \mathrm{H}, \mathrm{ArCH}_{3}\right), 2.22\left(\mathrm{~s}, 3 \mathrm{H}, \mathrm{CH}_{3}\right), 1.42\left(\mathrm{t}, 3 \mathrm{H}, J=7.1 \mathrm{~Hz}, \mathrm{OCH}_{2} \mathrm{CH}_{3}\right)$; ${ }^{13} \mathrm{C}-\mathrm{NMR}\left(101 \mathrm{MHz}, \mathrm{CDCl}_{3}\right): \delta 168.9,168.4,139.2,135.3,131.9,130.8,120.3,115.0,61.3,25.5,20.7,14.2$; HRMS (ESI): $\mathrm{m} / z$ [M + H] ${ }^{+}$calcd. for $\mathrm{C}_{12} \mathrm{H}_{15} \mathrm{NO}_{3}: 222.1130$, found: 222.1128 .

( \pm )2-Ethoxy-2-ethyl-6-methyl-1,2-dihydro-4H-benzo[d][1,3]oxazin-4-one (18b): $205 \mathrm{mg}$ (87\%, method 1) as white crystals, m.p. $78-79{ }^{\circ} \mathrm{C}$; IR (thin film): 3261, 1677, $1596 \mathrm{~cm}^{-1}$; ${ }^{1} \mathrm{H}-\mathrm{NMR}\left(400 \mathrm{MHz}, \mathrm{CDCl}_{3}\right): \delta 11.0$ (br s, 1H, NH), $8.62(\mathrm{~d}, 1 \mathrm{H}, J=8.6 \mathrm{~Hz}, \mathrm{ArH}), 7.83(\mathrm{~s}, 1 \mathrm{H}, \mathrm{ArH}), 7.34(\mathrm{~d}, 1 \mathrm{H}, J=8.6 \mathrm{~Hz}, \mathrm{ArH}), 4.37(\mathrm{q}, 2 \mathrm{H}$, $\left.J=7.0 \mathrm{~Hz}, \mathrm{OCH}_{2} \mathrm{CH}_{3}\right), 2.46\left(\mathrm{q}, 2 \mathrm{H}, J=7.5 \mathrm{~Hz}, \mathrm{CH}_{2} \mathrm{CH}_{3}\right), 2.33\left(\mathrm{~s}, 3 \mathrm{H}, \mathrm{ArCH}_{3}\right), 1.42(\mathrm{t}, 3 \mathrm{H}, J=7.0 \mathrm{~Hz}$, $\left.\mathrm{OCH}_{2} \mathrm{CH}_{3}\right), 1.27\left(\mathrm{t}, 3 \mathrm{H}, J=7.5 \mathrm{~Hz}, \mathrm{CH}_{2} \mathrm{CH}_{3}\right) ;{ }^{13} \mathrm{C}-\mathrm{NMR}\left(101 \mathrm{MHz}, \mathrm{CDCl}_{3}\right): \delta 172.7,168.4,139.4,135.3$, 131.7, 130.8, 120.3, 114.9, 61.3, 31.7, 20.7, 14.2, 9.7; HRMS (ESI): $\mathrm{m} / z$ [M + H] calcd. for $\mathrm{C}_{13} \mathrm{H}_{17} \mathrm{NO}_{3}$ : 236.1287, found: 236.1284 .

( \pm )-2-Ethoxy-6-methyl-2-propyl-1,2-dihydro-4H-benzo[d][1,3]oxazin-4-one (18c): $219 \mathrm{mg}$ (88\%, method 1) and $222 \mathrm{mg}$ (89\%, method 2) as off-white crystals, m.p. $39-40{ }^{\circ} \mathrm{C}$; IR (thin film): 3265, 1682, $1599 \mathrm{~cm}^{-1}$; 
${ }^{1} \mathrm{H}-\mathrm{NMR}\left(400 \mathrm{MHz}, \mathrm{CDCl}_{3}\right): \delta 10.98(\mathrm{br} \mathrm{s}, 1 \mathrm{H}, \mathrm{NH}), 8.62(\mathrm{~d}, 1 \mathrm{H}, J=8.6 \mathrm{~Hz}, \mathrm{ArH}), 7.83(\mathrm{~s}, 1 \mathrm{H}, \mathrm{ArH})$, $7.35(\mathrm{~d}, 1 \mathrm{H}, J=8.6 \mathrm{~Hz}, \mathrm{ArH}), 4.38\left(\mathrm{q}, 2 \mathrm{H}, J=7.1 \mathrm{~Hz}, \mathrm{OCH}_{2} \mathrm{CH}_{3}\right), 2.41\left(\mathrm{q}, 2 \mathrm{H}, J=7.5 \mathrm{~Hz}, \mathrm{CH}_{2} \mathrm{CH}_{2} \mathrm{CH}_{3}\right)$, $2.33\left(\mathrm{~s}, 3 \mathrm{H}, \mathrm{ArCH}_{3}\right), 1.78$ (sextet, $\left.2 \mathrm{H}, J=7.5 \mathrm{~Hz}, \mathrm{CH}_{2} \mathrm{CH}_{2} \mathrm{CH}_{3}\right), 1.42\left(\mathrm{t}, 3 \mathrm{H}, J=7.1 \mathrm{~Hz}, \mathrm{OCH}_{2} \mathrm{CH}_{3}\right.$ ), 1.01 $\left(\mathrm{t}, 3 \mathrm{H}, J=7.5 \mathrm{~Hz}, \mathrm{CH}_{2} \mathrm{CH}_{2} \mathrm{CH}_{3}\right) ;{ }^{13} \mathrm{C}-\mathrm{NMR}\left(101 \mathrm{MHz}, \mathrm{CDCl}_{3}\right): \delta 171.9,168.4,139.3,135.3,131.7,130.8$, $120.3,114.9,61.3,40.6,20.7,19.0,14.2,13.8$; HRMS (ESI): $\mathrm{m} / z[\mathrm{M}+\mathrm{H}]^{+}$calcd. for $\mathrm{C}_{14} \mathrm{H}_{19} \mathrm{NO}_{3}: 250.1443$, found: 250.1441 .

( \pm )-2-Ethoxy-6-methyl-2-phenyl-1,2-dihydro-4H-benzo[d][1,3]oxazin-4-one (18d): $249 \mathrm{mg}$ (88\%, method 1) as white crystals, m.p. $117-118^{\circ} \mathrm{C}$; IR (thin film): $3249,1662,1602 \mathrm{~cm}^{-1} ;{ }^{1} \mathrm{H}-\mathrm{NMR}\left(400 \mathrm{MHz}, \mathrm{CDCl}_{3}\right)$ : $\delta 11.98(\mathrm{br} \mathrm{s}, 1 \mathrm{H}, \mathrm{NH}), 8.82(\mathrm{~d}, 1 \mathrm{H}, J=8.5 \mathrm{~Hz}, \mathrm{ArH}), 8.05(\mathrm{~d}, 2 \mathrm{H}, J=6.8 \mathrm{~Hz}, \mathrm{ArH}), 7.89(\mathrm{~s}, 1 \mathrm{H}, \mathrm{ArH})$, 7.58-7.47 (complex, 3H), $7.42(\mathrm{~d}, 1 \mathrm{H}, J=8.5 \mathrm{~Hz}, \mathrm{ArH}), 4.42\left(\mathrm{q}, 2 \mathrm{H}, J=7.1 \mathrm{~Hz}, \mathrm{OCH}_{2} \mathrm{CH}_{3}\right), 3.36(\mathrm{~s}, 3 \mathrm{H}$, $\left.\mathrm{ArCH}_{3}\right), 1.44\left(\mathrm{q}, 3 \mathrm{H}, J=7.1 \mathrm{~Hz}, \mathrm{OCH}_{2} \mathrm{CH}_{3}\right) ;{ }^{13} \mathrm{C}-\mathrm{NMR}\left(101 \mathrm{MHz}, \mathrm{CDCl}_{3}\right): \delta 168.9,165.5,139.5,135.4$, 135.1, 132.1, 131.8, 131.0, 128.8, 127.3, 120.4, 115.4, 61.5, 20.8, 14.3; HRMS (ESI): $\mathrm{m} / z$ [M + H] ${ }^{+}$calcd. for $\mathrm{C}_{17} \mathrm{H}_{17} \mathrm{NO}_{3}$ : 284.1287, found: 284.1283 .

( \pm )-2-Ethoxy-2-methyl-7-nitro-1,2-dihydro-4H-benzo[d][1,3]oxazin-4-one (20a): $212 \mathrm{mg}$ (84\%, method 1) and $219 \mathrm{mg}(87 \%$, method 2$)$ as light tan crystals, m.p. $108-109^{\circ} \mathrm{C}$; IR (thin film): 3270, 1702, 1604, 1538, $1350 \mathrm{~cm}^{-1} ;{ }^{1} \mathrm{H}-\mathrm{NMR}\left(400 \mathrm{MHz}, \mathrm{CDCl}_{3}\right): \delta 11.16$ (br s, $\left.1 \mathrm{H}, \mathrm{NH}\right), 9.60(\mathrm{~d}, 1 \mathrm{H}, J=2.4 \mathrm{~Hz}, \mathrm{ArH}), 8.20$ $(\mathrm{d}, 1 \mathrm{H}, J=8.8 \mathrm{~Hz}, \mathrm{ArH}), 7.87(\mathrm{dd}, 1 \mathrm{H}, J=8.8,2.4 \mathrm{~Hz}, \mathrm{ArH}), 4.45\left(\mathrm{q}, 2 \mathrm{H}, J=7.1 \mathrm{~Hz}, \mathrm{OCH}_{2} \mathrm{CH}_{3}\right), 2.28(\mathrm{~s}$, $\left.3 \mathrm{H}, \mathrm{CH}_{3}\right), 1.46\left(\mathrm{t}, 3 \mathrm{H}, \mathrm{J}=7.1 \mathrm{~Hz}, \mathrm{OCH}_{2} \mathrm{CH}_{3}\right) ;{ }^{13} \mathrm{C}-\mathrm{NMR}\left(101 \mathrm{MHz}, \mathrm{CDCl}_{3}\right): \delta 169.2,166.9,151.2,142.2$, 131.9, 119.3, 116.4, 115.2, 62.5, 25.5, 14.1; HRMS (ESI): $m / z[\mathrm{M}+\mathrm{H}]^{+}$calcd. for $\mathrm{C}_{11} \mathrm{H}_{12} \mathrm{~N}_{2} \mathrm{O}_{5}: 253.0825$, found: 253.0824 .

( \pm )-2-Ethoxy-2-ethyl-7-nitro-1,2-dihydro-4H-benzo[d][1,3]oxazin-4-one (20b): $229 \mathrm{mg}$ (86\%, method 1) and $234 \mathrm{mg}\left(88 \%\right.$, method 2) as light tan crystals, m.p. $74-75^{\circ} \mathrm{C}$; IR (thin film): 3273, 1694, 1600, 1538, $1349 \mathrm{~cm}^{-1} ;{ }^{1} \mathrm{H}-\mathrm{NMR}\left(400 \mathrm{MHz}, \mathrm{CDCl}_{3}\right): \delta 11.18$ (br s, $\left.1 \mathrm{H}, \mathrm{NH}\right), 9.65(\mathrm{~d}, 1 \mathrm{H}, J=2.4 \mathrm{~Hz}, \mathrm{ArH}), 8.20(\mathrm{~d}$, $1 \mathrm{H}, J=8.8 \mathrm{~Hz}, \mathrm{ArH}), 7.86(\mathrm{dd}, 1 \mathrm{H}, J=8.8,2.4 \mathrm{~Hz}, \mathrm{ArH}), 4.45\left(\mathrm{q}, 2 \mathrm{H}, J=7.1 \mathrm{~Hz}, \mathrm{OCH}_{2} \mathrm{CH}_{3}\right), 2.53(\mathrm{q}, 2 \mathrm{H}$, $\left.J=7.5 \mathrm{~Hz}, \mathrm{CH}_{2} \mathrm{CH}_{3}\right), 1.46\left(\mathrm{t}, 3 \mathrm{H}, J=7.1 \mathrm{~Hz}, \mathrm{OCH}_{2} \mathrm{CH}_{3}\right), 1.30\left(\mathrm{t}, 3 \mathrm{H}, J=7.5 \mathrm{~Hz}, \mathrm{CH}_{2} \mathrm{CH}_{3}\right) ;{ }^{13} \mathrm{C}-\mathrm{NMR}$ (101 MHz, $\left.\mathrm{CDCl}_{3}\right): \delta 173.1,166.9,151.2,142.5,131.9,119.3,116.3,115.3,62.4,31.6,14.1,9.3 ;$ HRMS (ESI): $m / z[\mathrm{M}+\mathrm{H}]^{+}$calcd. for $\mathrm{C}_{12} \mathrm{H}_{14} \mathrm{~N}_{2} \mathrm{O}_{5}: 267.0981$, found: 267.0977 .

( \pm )-2-Ethoxy-2-propyl-7-nitro-1,2-dihydro-4H-benzo[d][1,3]oxazin-4-one (20c): $238 \mathrm{mg}$ (85\%, method 1) and $245 \mathrm{mg}$ (88\%, method 2) as light tan crystals, m.p. $66-67^{\circ} \mathrm{C}$; IR (thin film): 3270, 1697, 1604, 1538, $1349 \mathrm{~cm}^{-1},{ }^{1} \mathrm{H}-\mathrm{NMR}\left(400 \mathrm{MHz}, \mathrm{CDCl}_{3}\right): \delta 11.17$ (br s, $\left.1 \mathrm{H}, \mathrm{NH}\right), 9.65(\mathrm{~d}, 1 \mathrm{H}, J=2.4 \mathrm{~Hz}, \mathrm{ArH}), 8.20(\mathrm{~d}$, $1 \mathrm{H}, J=8.8 \mathrm{~Hz}, \mathrm{ArH}), 7.86(\mathrm{dd}, 1 \mathrm{H}, J=8.8,2.4 \mathrm{~Hz}, \mathrm{ArH}), 4.45\left(\mathrm{q}, 2 \mathrm{H}, J=7.1 \mathrm{~Hz}, \mathrm{OCH}_{2} \mathrm{CH}_{3}\right), 2.47(\mathrm{q}, 2 \mathrm{H}$, $J=7.4 \mathrm{~Hz}, \mathrm{CH}_{2} \mathrm{CH}_{2} \mathrm{CH}_{3}$ ), 1.81 (sextet, $\left.2 \mathrm{H}, J=7.4 \mathrm{~Hz}, \mathrm{CH}_{2} \mathrm{CH}_{2} \mathrm{CH}_{3}\right), 1.46\left(\mathrm{t}, 3 \mathrm{H}, J=7.1 \mathrm{~Hz}, \mathrm{OCH}_{2} \mathrm{CH}_{3}\right.$ ), $1.04\left(\mathrm{t}, 3 \mathrm{H}, \mathrm{J}=7.4 \mathrm{~Hz}, \mathrm{CH}_{2} \mathrm{CH}_{2} \mathrm{CH}_{3}\right) ;{ }^{13} \mathrm{C}-\mathrm{NMR}\left(101 \mathrm{MHz}, \mathrm{CDCl}_{3}\right): \delta 172.3,166.9,151.2,142.5,131.9$, $119.3,116.3,115.3,62.4,40.4,18.8,14.1,13.7$; HRMS (ESI): $\mathrm{m} / z[\mathrm{M}+\mathrm{H}]^{+}$calcd. for $\mathrm{C}_{13} \mathrm{H}_{16} \mathrm{~N}_{2} \mathrm{O}_{5}$ : 281.1138, found: 281.1140 .

( \pm )-2-Ethoxy-7-nitro-2-phenyl-1,2-dihydro-4H-benzo[d][1,3]oxazin-4-one (20d): $283 \mathrm{mg}$ (90\%, method 1) as off-white crystals, m.p. $180-18{ }^{\circ} \mathrm{C}$; IR (thin film): $3257,1673,1651,1600,1538,1346 \mathrm{~cm}^{-1} ;{ }^{1} \mathrm{H}-\mathrm{NMR}$ $\left(400 \mathrm{MHz}, \mathrm{CDCl}_{3}\right): \delta 11.62$ (br s, $\left.1 \mathrm{H}, \mathrm{NH}\right), 9.31(\mathrm{~d}, 1 \mathrm{H}, J=2.4 \mathrm{~Hz}, \mathrm{ArH}), 8.20(\mathrm{~d}, 1 \mathrm{H}, J=8.8 \mathrm{~Hz}, \mathrm{ArH})$, $8.03(\mathrm{dd}, 1 \mathrm{H}, J=8.8,2.4 \mathrm{~Hz}, \mathrm{ArH}), 7.97(\mathrm{~d}, 2 \mathrm{H}, J=7.4 \mathrm{~Hz}, \mathrm{ArH}, 7.69(\mathrm{t}, 1 \mathrm{H}, J=7.4 \mathrm{~Hz}, \mathrm{ArH}), 7.61(\mathrm{t}$, $2 \mathrm{H}, J=7.4 \mathrm{~Hz}, \mathrm{ArH}), 4.38\left(\mathrm{q}, 2 \mathrm{H}, J=7.1 \mathrm{~Hz}, \mathrm{OCH}_{2} \mathrm{CH}_{3}\right), 1.33\left(\mathrm{t}, 3 \mathrm{H}, J=7.1 \mathrm{~Hz}, \mathrm{OCH}_{2} \mathrm{CH}_{3}\right) ;{ }^{13} \mathrm{C}-\mathrm{NMR}$ $\left(101 \mathrm{MHz}, \mathrm{CDCl}_{3}\right): \delta 166.7,165.7,150.6,141.0,134.1,133.1,132.7,129.5,127.7,1239,118.2,116.0,62.6$, 14.3; HRMS (ESI): $m / z$ [M + H] $]^{+}$calcd. for $\mathrm{C}_{16} \mathrm{H}_{14} \mathrm{~N}_{2} \mathrm{O}_{5}$ : 315.0981, found: 315.0975.

( \pm )-7-Chloro-2-ethoxy-2-methyl-1,2-dihydro-4H-benzo[d][1,3]oxazin-4-one (21a): $203 \mathrm{mg}$ (84\%, method 1) as white crystals, m.p. $75-76{ }^{\circ} \mathrm{C}$; IR (thin film): $3262,1681,1578 \mathrm{~cm}^{-1} ;{ }^{1} \mathrm{H}-\mathrm{NMR}\left(400 \mathrm{MHz}, \mathrm{CDCl}_{3}\right): \delta$ $10.70(\mathrm{br} \mathrm{s}, 1 \mathrm{H}, \mathrm{NH}), 8.43(\mathrm{~d}, 1 \mathrm{H}, J=2.2 \mathrm{~Hz}, \mathrm{ArH}), 7.92(\mathrm{~d}, 1 \mathrm{H}, J=8.6 \mathrm{~Hz}, \mathrm{ArH}), 7.23(\mathrm{dd}, 1 \mathrm{H}, J=8.6$, $2.2 \mathrm{~Hz}, \mathrm{ArH}), 4.83\left(\mathrm{q}, 2 \mathrm{H}, J=7.1 \mathrm{~Hz}, \mathrm{OCH}_{2} \mathrm{CH}_{3}\right), 2.15\left(\mathrm{~s}, 3 \mathrm{H}, \mathrm{CH}_{3}\right), 1.34\left(\mathrm{t}, 3 \mathrm{H}, J=7.1 \mathrm{~Hz}, \mathrm{OCH}_{2} \mathrm{CH}_{3}\right)$; ${ }^{13} \mathrm{C}-\mathrm{NMR}\left(101 \mathrm{MHz}, \mathrm{CDCl}_{3}\right): \delta 169.1,167.7,142.4,140.8,131.8,122.6,120.1,113.2,61.6,25.5,14.2$; HRMS (ESI): $m / z[\mathrm{M}+\mathrm{H}]^{+}$calcd. for $\mathrm{C}_{11} \mathrm{H}_{12}{ }^{35} \mathrm{ClNO}_{3}: 242.0584$, found: 242.0582 . 
(土)-7-Chloro-2-ethoxy-2-ethyl-1,2-dihydro-4H-benzo[d][1,3]oxazin-4-one (21b): $215 \mathrm{mg}(84 \%$, method 1) and $220 \mathrm{mg}(86 \%$, method 2$)$ as white crystals, m.p. $69-70{ }^{\circ} \mathrm{C}$; IR (thin film): 3238, 1685, $1581 \mathrm{~cm}^{-1}$; ${ }^{1} \mathrm{H}-\mathrm{NMR}\left(400 \mathrm{MHz}, \mathrm{CDCl}_{3}\right): \delta 11.17$ (br s, 1H, NH), $8.86(\mathrm{~d}, 1 \mathrm{H}, \mathrm{J}=2.2 \mathrm{~Hz}, \mathrm{ArH}), 7.96(\mathrm{~d}, 1 \mathrm{H}, \mathrm{J}=8.6 \mathrm{~Hz}$, $\mathrm{ArH}), 7.04(\mathrm{dd}, 1 \mathrm{H}, J=8.6,2.2 \mathrm{~Hz}, \mathrm{ArH}), 4.38\left(\mathrm{q}, 2 \mathrm{H}, J=7.1 \mathrm{~Hz}, \mathrm{OCH}_{2} \mathrm{CH}_{3}\right), 2.48(\mathrm{q}, 2 \mathrm{H}, J=7.5 \mathrm{~Hz}$, $\left.\mathrm{CH}_{2} \mathrm{CH}_{3}\right), 1.41\left(\mathrm{t}, 3 \mathrm{H}, \mathrm{J}=7.1 \mathrm{~Hz}, \mathrm{OCH}_{2} \mathrm{CH}_{3}\right), 1.27\left(\mathrm{t}, 3 \mathrm{H}, \mathrm{J}=7.5 \mathrm{~Hz}, \mathrm{CH}_{2} \mathrm{CH}_{3}\right) ;{ }^{13} \mathrm{C}-\mathrm{NMR}(101 \mathrm{MHz}$, $\left.\mathrm{CDCl}_{3}\right): \delta 173.0,167.8,142.6,140.9,131.8,122.5,120.2,113.2,61.6,31.7,14.2,9.5 ; \mathrm{HRMS}(\mathrm{ESI}): \mathrm{m} / z$ [M + $\mathrm{H}^{+}$calcd. for $\mathrm{C}_{12} \mathrm{H}_{14}{ }^{35} \mathrm{ClNO}_{3}: 256.0741$, found: 256.0743 .

(士)-7-Chloro-2-ethoxy-2-propyl-1,2-dihydro-4H-benzo[d][1,3]oxazin-4-one (21c): $224 \mathrm{mg}(83 \%$, method 1) and $227 \mathrm{mg}\left(84 \%\right.$, method 2) as white semisolid, m.p. $25-26{ }^{\circ} \mathrm{C}$; IR (thin film): 3280, 1691, 1581 $\mathrm{cm}^{-1}$; ${ }^{1} \mathrm{H}-\mathrm{NMR}\left(400 \mathrm{MHz}, \mathrm{CDCl}_{3}\right): \delta 11.16$ (br s, 1H, NH), 8.85 (s, 1H, ArH), $7.96(\mathrm{~d}, 1 \mathrm{H}, J=8.6 \mathrm{~Hz}, \mathrm{ArH}), 7.04$ $(\mathrm{dd}, 1 \mathrm{H}, J=8.6,2.2 \mathrm{~Hz}, \mathrm{ArH}), 4.38\left(\mathrm{q}, 2 \mathrm{H}, J=7.1 \mathrm{~Hz}, \mathrm{OCH}_{2} \mathrm{CH}_{3}\right), 2.42\left(\mathrm{q}, 2 \mathrm{H}, J=7.4 \mathrm{~Hz}, \mathrm{CH}_{2} \mathrm{CH}_{2} \mathrm{CH}_{3}\right)$, 1.78 (sextet, $\left.2 \mathrm{H}, J=7.4 \mathrm{~Hz}, \mathrm{CH}_{2} \mathrm{CH}_{2} \mathrm{CH}_{3}\right), 1.42\left(\mathrm{t}, 3 \mathrm{H}, J=7.1 \mathrm{~Hz}, \mathrm{OCH}_{2} \mathrm{CH}_{3}\right), 1.02(\mathrm{t}, 3 \mathrm{H}, J=7.4 \mathrm{~Hz}$, $\left.\mathrm{CH}_{2} \mathrm{CH}_{2} \mathrm{CH}_{3}\right) ;{ }^{13} \mathrm{C}-\mathrm{NMR}\left(101 \mathrm{MHz}, \mathrm{CDCl}_{3}\right): \delta 172.2,167.7,142.5,140.8,131.8,122.5,120.2,113.2,61.6$, 40.5, 18.9, 14.2, 13.2; HRMS (ESI): $m / z[\mathrm{M}+\mathrm{H}]^{+}$calcd. for $\mathrm{C}_{13} \mathrm{H}_{16}{ }^{35} \mathrm{ClNO}_{3}$ : 270.0897, found: 270.0893 .

(土)-7-Chloro-2-ethoxy-2-phenyl-1,2-dihydro-4H-benzo[d][1,3]oxazin-4-one (21d): $258 \mathrm{mg}$ (85\%, method 1) as white crystals, m.p. $128-129{ }^{\circ} \mathrm{C}$; IR (thin film): 3241, 1673, $1585 \mathrm{~cm}^{-1}$; ${ }^{1} \mathrm{H}-\mathrm{NMR}(400 \mathrm{MHz}$, $\mathrm{CDCl}_{3}$ ): $\delta 12.13$ (br s, 1H, NH), $9.05(\mathrm{~d}, 1 \mathrm{H}, J=2.1 \mathrm{~Hz}, \mathrm{ArH}), 8.03$ (overlapping signals, apparent $\mathrm{t}$, $3 \mathrm{H}, \mathrm{J} \approx 8.4 \mathrm{~Hz}, \mathrm{ArH}), 7.60-7.49$ (complex, 3H, $\mathrm{ArH}$ ), 7.08 (dd, 1H, J = 8.6, $2.1 \mathrm{~Hz}, \mathrm{ArH}), 4.42(\mathrm{q}, 2 \mathrm{H}$, $\left.J=7.1 \mathrm{~Hz}, \mathrm{OCH}_{2} \mathrm{CH}_{3}\right), 1.43\left(\mathrm{t}, 3 \mathrm{H}, J=7.1 \mathrm{~Hz}, \mathrm{OCH}_{2} \mathrm{CH}_{3}\right) ;{ }^{13} \mathrm{C}-\mathrm{NMR}\left(101 \mathrm{MHz}, \mathrm{CDCl}_{3}\right): \delta 168.1,165.7$, 142.7, 141.0, 134.4, 132.2, 132.0, 128.9, 127.4, 122.8, 120.3, 113.6, 61.8, 14.2; HRMS (ESI): $m / z$ [M + H] ${ }^{+}$ calcd. for $\mathrm{C}_{16} \mathrm{H}_{14}{ }^{35} \mathrm{ClNO}_{3}$ : 304.0741, found: 304.0737 .

(土)-2-Ethoxy-2-methyl-1,2-dihydro-4H-pyrido[2,3-d][1,3]oxazin-4-one (22a): $177 \mathrm{mg}(85 \%$, method 1) as white crystals, m.p. $55-56{ }^{\circ} \mathrm{C}$; IR (thin film): 3270, 1680, $1589 \mathrm{~cm}^{-1} ;{ }^{1} \mathrm{H}-\mathrm{NMR}\left(400 \mathrm{MHz}, \mathrm{CDCl}_{3}\right): \delta$ 10.79 (br s, 1H, NH), 8.59 (dd, 1H, J = 4.8, 2.0 Hz, PyH), 8.33 (dd, 1H, J = 7.9, 2.0 Hz, PyH), 7.06 (dd, $1 \mathrm{H}, J=7.9,4.8 \mathrm{~Hz}, \mathrm{PyH}), 4.41\left(\mathrm{q}, 2 \mathrm{H}, J=2.1 \mathrm{~Hz}, \mathrm{OCH}_{2} \mathrm{CH}_{3}\right), 2.42\left(\mathrm{~s}, 3 \mathrm{H}, \mathrm{CH}_{3}\right), 1.42(\mathrm{t}, 3 \mathrm{H}, J=7.1 \mathrm{~Hz}$, $\left.\mathrm{OCH}_{2} \mathrm{CH}_{3}\right) ;{ }^{13} \mathrm{C}-\mathrm{NMR}\left(101 \mathrm{MHz}, \mathrm{CDCl}_{3}\right): \delta 169.6,166.6,152.9,152.5,140.0,118.0,111.1,62.0,26.0,14.2$; HRMS (ESI): $m / z[M+H]^{+}$calcd. for $\mathrm{C}_{10} \mathrm{H}_{12} \mathrm{~N}_{2} \mathrm{O}_{3}: 209.0926$, found: 209.0925 .

\section{Conclusions}

We have studied the formation of $4 H$-benzo[d][1,3]oxazin-4-ones under thermal conditions with acetic acid as well as microwave conditions without acid. In most cases, the target heterocycle was formed in high yield. The reaction was performed with no solvent, and the desired products were isolated by crystallization and trituration. The microwave reaction proceeded much faster $(0.75-3 \mathrm{~h})$ than the thermal reactions (4-48 h) and did not require added acid. Thus, the microwave protocol represents the superior procedure. Stereoelectronic effects strongly impacted the outcome of the reaction. In addition to the benzoxazinone products, a dihydro intermediate resulting from failure to undergo the final elimination was observed in some substrates. The formation of the dihydro compound arose in reactants where the fused aromatic ring possessed a second electron-withdrawing group in addition to the conjugated carbonyl on the ortho carbon. This could deactivate the electron pair on N1 and slow the elimination process, even if the C2 ethoxy group is able to adopt the required pseudo-axial conformation. The formation of these dihydro systems provides access to a new substitution pattern in this family of compounds, and could yield additional candidates for biological testing. This finding coupled with the fact that orthoesters are easily prepared from nitriles $[17,18]$ should provide a route to many members of this novel class of structures.

Supplementary Materials: The supplemental materials are available online.

Author Contributions: J.K.A-G. did the experimental work. R.A.B. directed the project, checked the data, and wrote the paper. Each of the authors read and approved the final version of the manuscript before submission.

Funding: This research was funded, in part, by the NSF MRI Program (BIR-9512269). 
Acknowledgments: J.K.A-G. wishes to thank the Oklahoma State University (OSU) Department of Chemistry for a teaching assistantship. The authors are also grateful for support from the NSF (BIR-9512269), the Oklahoma State Regents for Higher Education, the W. M. Keck Foundation, and Conoco, Inc. that established the Oklahoma State-wide NMR facility. The authors also thank the OSU College of Arts and Sciences for funds that allowed the Chemistry Department to purchase a new $400 \mathrm{MHz}$ NMR for this facility.

Conflicts of Interest: The authors declare no conflict of interest.

\section{References}

1. Gavin, J.T.; Annor-Gyamfi, J.K.; Bunce, R.A. Quinazolin-4(3H)-ones and 5,6-dihydropyrimidin-4(3H)-ones from $\beta$-aminoamides and orthoesters. Molecules 2018, 23, 2925. [CrossRef] [PubMed]

2. Fenton, G.; Newton, C.G.; Wyman, B.M.; Bagge, P.; Dron, D.I.; Riddell, D.; Jones, G.D. Hypolipidemic 2-[4-(1,1-dimethylethyl)phenyl-4H-3,1-benzoxazin-4-ones. Structure activity relationships of a novel series of high density lipoprotein elevators. J. Med. Chem. 1989, 32, 265-272. [CrossRef] [PubMed]

3. Krantz, A.; Spencer, R.W.; Tam, T.F.; Liak, T.J.; Copp, L.J.; Thomas, E.M.; Rafferty, S.P. Design and synthesis of 4H-3,1-benzoxazin-4-ones as potent alternate substrate inhibitors of human leukocyte elastase. J. Med. Chem. 1990, 33, 464-479. [CrossRef] [PubMed]

4. Goel, P.; Jumpertz, T.; Tichá, A.; Ogorek, I.; Mikles, D.C.; Hubalek, M.; Piertrzik, C.U.; Strisovsky, K.; Schmidt, B.; Weggen, S. Discovery and validation of 2-styryl substituted benzoxazin-4-ones as a novel scaffold for rhomboid protease inhibitors. Bioorg. Med. Chem. Lett. 2018, 28, 1417-1422. [CrossRef] [PubMed]

5. Yang, J.; Barniol-Xicota, M.; Nguyen, M.T.N.; Ticha, A.; Strisovsky, K.; Verhelst, S.H.L. Benzoxazin-4-ones as novel, easily accessible inhibitors for rhomboid proteases. Bioorg. Med. Chem. Lett. 2018, 28, 1423-1427. [CrossRef] [PubMed]

6. Madkour, H.M.F. Reactivity of 4H-3,1-benzoxazin-4-ones towards nitrogen and carbon nucleophilic reagents: Applications to the synthesis of new heterocycles. ARKIVOC 2004, 1, 36-54.

7. Baravkar, S.B.; Roy, A.; Gawade, R.L.; Puranik, V.G.; Sanjayan, G.J. Nucleophilic ring opening of benzoxazinone by DBU: Some observations. Synth. Commun. 2014, 44, 2955-2960. [CrossRef]

8. Zentmeyer, D.T.; Wagner, E.C. The so-called acylanthranils (3,1,4-benzoxazones). I. preparation; reactions with water, ammonia and aniline; structure. J. Org. Chem. 1949, 14, 967-981. [CrossRef]

9. Bain, D.I.; Smalley, R.K. Synthesis of 2-substituted-4H-3,1-benzoxazin-4-ones. J. Chem. Soc. C $1968,1593$. [CrossRef]

10. Larksarp, C.; Alper, H. A simple synthesis of 2-substituted 4H-3,1-benzoxazin-4-ones by palladium-catalyzed cyclocarbonylation of $o$-iodoanilines with acid chlorides. Org. Lett. 1999, 1, 1619-1622. [CrossRef]

11. Yamashita, M.; Iida, A. One-pot approach to 2-arylbenzoxazinone derivatives from 1-alkynylanilines using copper mediated tandem reactions. Tetrahedron 2014, 70, 5746-5751. [CrossRef]

12. Shang, X.-X.; Vu, H.-M.; Li, X.-Q. One-pot synthesis of 2-arylbenzoxazinones from 2-arylindoles with (diacetoxyiodo)benzene as the sole oxidant. Synthesis 2018, 50, 377-383.

13. Plescia, S.; Raffa, D.; Plescia, F.; Casula, G.; Maggio, B.; Daidone, G.; Raimondi, M.V.; Cuismano, M.G.; Bombieri, G.; Meneghetti, F. Synthesis and biological evaluation of new indazole derivatives. ARKIVOC 2010, 10, 163-177.

14. Deslongchamps, P. Stereoelectronic Effects in Organic Chemistry; Pergamon Press: Oxford, UK, 1983; pp. 4-53.

15. Smith, M.B.; March, J. March's Advanced Organic Chemistry, 6th ed.; Wiley-Interscience: New York, NY, USA, 2007; pp. 204-206.

16. Kleinpeter, E.; Thielemann, J. Synthesis and conformational analysis of mono- and trans-1,4-dialkoxy substituted cyclohexanes-steric substituent/skeleton interactions. Tetrahedron 2007, 63, 9071-9081. [CrossRef]

17. McElvain, S.N.; Nelson, J.W. The preparation of orthesters. J. Am. Chem. Soc. 1942, 64, 1825-1827. [CrossRef]

18. Noe, M.; Perosa, A.; Selva, M. A flexible Pinner preparation of orthoesters: The model case of trimethylorthobenzoate. Green Chem. 2013, 15, 2252-2260. [CrossRef]

Sample Availability: Samples of the compounds are not available from the authors.

(C) 2019 by the authors. Licensee MDPI, Basel, Switzerland. This article is an open access article distributed under the terms and conditions of the Creative Commons Attribution (CC BY) license (http://creativecommons.org/licenses/by/4.0/). 